\title{
The shifted focus of interest in the temperature-blood pressure relationship: from load to variability
}

\author{
Pietro Amedeo Modesti ${ }^{1,2}$
}

Received: 23 July 2021 / Accepted: 1 August 2021 / Published online: 3 September 2021

(c) The Japanese Society of Hypertension 2021

The contribution of Umishio et al. [1] is an important step forward for at least three reasons: first, data clearly show that morning-evening blood pressure (BP) difference, a measurement of BP variability that can easily be selfmeasured at home, is closely related to the variation in temperature, thus moving the focus from Ambulatory Blood Pressure Monitoring (ABPM) to Home Blood Pressure (HBP) monitoring; second, the prevailing effect of indoor over outdoor temperature in inducing changes in $\mathrm{BP}$ variability clearly emerges; and third, the project will offer the government of Japan a measure of the health effects of insulation retrofitting, a structural housing intervention. The Smart Wellness Housing (SWH) project is indeed aimed at quantitatively evaluating the relationship between indoor temperature and home BP (HBP) in Japan among participants who lived in houses that did not meet the $S$ (Supreme) standards of the "Act on the Promotion of Dissemination of Long-Lasting Quality Housing" in Japan and decided to conduct insulation retrofitting. Therefore, the present data can be considered the baseline observation of a national intervention for health promotion in Japan. Cold housing, below the World Health Organization's recommended minimum of $18{ }^{\circ} \mathrm{C}$ [2], has been associated with avoidable excess winter mortality [3]. Interventions involving insulation retrofitting have been performed at national levels in several countries worldwide. Moreover, recently, the EU Commission included funds for renovation of housing insulation in "The Recovery and Resilience Facility" project. Although health researchers built a solid foundation for housing interventions, they rarely followed the projects very closely, and the measures of efficacy on health promotion

Pietro Amedeo Modesti

pamodesti@unifi.it

1 Department of Experimental and Clinical Medicine, University of Florence, Florence, Italy

2 Azienda Ospedaliero-Universitaria Careggi, Florence, Italy are limited [4]. The study is therefore a sign of the great work carried out by Japanese clinical researchers in the field of climate health in recent years.

The clinical implications of changes in BP in response to changes in environmental temperature have gradually emerged over the last few decades.

\section{Climate, a barrier to baseline BP estimation}

The relationship between ambient temperature and BP has been considered for many years as little more than a curiosity; physicians only saw it as something useful for the assessment of basal (office) blood pressure, and office environment standardization was the only possibility for the temperature to stay within BP guidelines. Should the patient come from a colder environment, it was advisable to wait for the patient to reach the optimal temperature $\left(22-24{ }^{\circ} \mathrm{C}\right)$ before carrying out the evaluation. How temperature influences BP outside the clinic was not important to physicians, seeming rather of interest only for physiologists. The guidelines remained focused on finding ways to obtain a BP evaluation as "static" as possible, while BP is one of the most dynamic vital parameters.

In the era of controlled clinical trials, the influence of seasonal BP changes started to emerge. Seasonal BP variation parallels seasonal changes in environmental temperature, with lower BP levels in summer and higher BPs at lower temperatures in winter. Seasonal variations could thus mask the demonstration of a drug's efficacy in a controlled clinical study that, having begun enrollment in summer, ended in winter. This observation was not enough to divert the researchers' attention away from the search for stability, although the negative relationship between outdoor temperature and BP values was consistently observed even when measurements were taken in comfortably warm rooms [5]. This variation was independent of anthropometric data and baseline BP values but was related to the participants' age. Variations in BP were indeed greater in older people 
than in younger participants. The implications for treatment have received interest from physicians.

\section{Climate changes and BP treatment}

Treated hypertensive patients with controlled BP in summer may show a considerable BP rise above the recommended threshold in winter, requiring up-titration of drug treatment. On the other hand, as physicians, we often have to be cautious about treatment titration when dealing with hypertensive patients with heart failure who are receiving diuretics and Ang II antagonists. In this context, kidney perfusion is highly blood pressure dependent, and if diuretic and antihypertensive therapy are not adapted in summer, the risk of acute renal failure increases, especially in elderly patients. Great attention has been given to ABPM. First, the introduction of ABPM into clinical practice has started to attract the attention of clinicians to the clinical implications of the external environment. Evidence has begun to emerge showing the importance of adapting antihypertensive treatment to daily and seasonal variations; the decision of the time of day a drug should be used by a patient or the decision to reduce BP treatment in summer could be supported, although the issue has not been considered specifically in hypertension guidelines. Second, attention has started to move toward identifying determinants of survival or cerebrovascular events related to BP load and BP variability. A Japanese group highlighted the close relationship between cerebrovascular events and night/day or better night/morning variation in $\mathrm{BP}$ with the introduction of the so-called morning surge [6]. Moreover, refinements led to the identification of the preferential role of indoor temperature in determining BP changes. After all, people spend a large part of their time indoors, and it is natural to think that it is the indoor rather than the outdoor temperature that exerts the prevailing influence on arterial pressure [7]. BP variations are naturally accentuated, if not determined, by temperature variations. Increased BP variability was found to be associated with an increased risk of $\mathrm{CV}$ events. This is reminiscent of the increased risk that was found to be linked to an increase in systolic pressure, independent of an increase in diastolic pressure, and hence to an increase in differential BP. Of course, increased variability in response to temperature, increased differential pressure, and an increased conduction velocity are all markers of vascular aging, with increased arterial wall stiffness. Most importantly, what intervention can be implemented to reduce BP variability and will an intervention result in the reduction of cardiovascular outcomes? An answer will be provided by the Smart Wellness Housing (SWH) project [1].

\section{Climate, BP variability and housing}

Strategies identified to combat the health effects of temperature changes are mainly aimed at reducing the effects of low temperature. The simplest strategy is on a personal level, by adapting clothing, while the second, of a structural type, concerns housing interventions. Policies and measures have been promoted to increase the efficient use of energy indoors, along with advice suggesting that citizens wear appropriate warm protective clothing and keep active outdoors. The social implications of these recommendations are relevant because the health consequences of climate changes are most likely to affect individuals in the lowest socioeconomic strata of the population living in poor housing conditions. The search for the basic BP value is no longer the only focal topic in the context of temperature, and temperature-induced BP variations are now considered by supernational institutional health promoters (WHO) and political decision-makers (such as in England and Japan). Important initiatives have been launched, and great attention is now placed on housing. In Europe, excess winter mortality was found to be higher in Southern Europe (Portugal and Spain) than in Scandinavia and Northern Europe (Finland and Germany) [8]. In Europe, the thermal efficiency of housing, as well as the capability to cope with cold weather, were indeed found to increase with rising latitude [9]. In the reanalysis of $\mathrm{BP}$ data collected in the WHO MONICA Project [10], the random effects that seasons had on the main risk factor for CV events (BP) were latitude-dependent, being lower for countries with colder climates and higher for countries with warmer climates.

Umishio's work [1] elucidates why these two new approaches were born in Japan and how much the temperature-blood pressure relationship can have different implications in different countries. Lifestyle factors, and consequently the characteristics of buildings, make housing more strongly linked in the climate-health relationship in Japan than in Europe or America. Houses have less effective environmental insulation, and exposure to climate changes is relevant.

In Japan, this is independent of socioeconomic status and is not accompanied by a reduced ability to heat homes but perhaps rather by greater energy consumption. The thermal insulation of homes and the relationship between housing and health have recently been addressed by the WHO [2] The WHO is interested in fighting the consequences of harsher winters both in countries and in social groups that are unable to provide heating in homes due to low incomes. In high-income countries, interventions are generally seen as a function to improve energy efficiency. For low-income countries, for the poorest segments of the world population, 
and for populations fleeing from wars and currently hosted in refugee camps in many areas of Europe, these interventions could instead translate into a reduction in strokes during the winter months. The data that will be collected in Japan will also be of great use in the context of these humanitarian emergencies.

\section{Compliance with ethical standards}

Conflict of interest The authors declare no competing interests.

Publisher's note Springer Nature remains neutral with regard to jurisdictional claims in published maps and institutional affiliations.

\section{References}

1. Umishio W, Ikaga T, Kario K, Fujino Y, Suzuki M, et al. Impact of indoor temperature instability on diurnal and day-by-day variability of home blood pressure in winter: A Nationwide Smart Wellness Housing Survey in Japan. Hypertens Res. 2021.

2. WHO Housing and health guidelines. Geneva: World Health Organization; 2018. Licence: CC BY-NC-SA 3.0 IGO.
3. Fowler T, Southgate RJ, Waite T, Harrell R, Kovats S, Bone A, et al. Excess winter deaths in Europe: a multi-country descriptive analysis. Eur J Public Health. 2015;25:339-45.

4. Fyfe C, Telfar L, Barnard, Howden-Chapman P, Douwes J. Association between home insulation and hospital admission rates: retrospective cohort study using linked data from a national intervention programme. BMJ. 2020;371:m4571.

5. Modesti PA. Season, temperature and blood pressure: a complex interaction. Eur J Intern Med. 2013;24:604-7.

6. Kario K, Pickering TG, Umeda Y, Hoshide S, Hoshide Y, Morinari $\mathrm{M}$, et al. Morning surge in blood pressure as a predictor of silent and clinical cerebrovascular disease in elderly hypertensives: a prospective study. Circulation. 2003;107:1401-6.

7. Modesti PA, Morabito M, Massetti L, Rapi S, Orlandini S, Mancia G, et al. Seasonal blood pressure changes: an independent relationship with temperature and daylight hours. Hypertension. 2013;61:908-14.

8. Healy JD. Excess winter mortality in Europe: a cross country analysis identifying key risk factors. J Epidemiol Community Health. 2003;57:784-9.

9. The Eurowinter Group. Cold exposure and winter mortality from ischaemic heart disease, cerebrovascular disease, respiratory disease, and all causes in warm and cold regions of Europe. The Eurowinter Group. Lancet. 1997;349:1341-6.

10. Barnett AG, Sans S, Salomaa V, Kuulasmaa K, Dobson AJ, Project WM. The effect of temperature on systolic blood pressure. Blood Press Monit. 2007;12:195-203. 